\title{
Investigation of the forest-fire model on a small-world network
}

\author{
I. Graham* and C. C. Matthai \\ Department of Physics and Astronomy, University of Wales, Cardiff, United Kingdom \\ (Received 17 September 2002; revised manuscript received 29 April 2003; published 12 September 2003)

\begin{abstract}
It is shown that the forest-fire model of Bak et al. run on a square lattice network with additional long-range interactions in the spirit of a small-world network results in a scale-free system reminiscent of self-organized criticality without recourse to fine tuning. As the number of these long-range interactions is increased, the cluster size distribution exponent is found to decrease in magnitude as the small-world regime is entered, indicating a change in its universality class. It is suggested that such a model could have applicability in the study of disease spreading in human populations.
\end{abstract}

DOI: 10.1103/PhysRevE.68.036109

PACS number(s): 05.65.+b, 87.23.Ge, 89.75.Da

\section{INTRODUCTION}

Over a decade ago Bak et al. [1] proposed a lattice model, which through the emergence of complex, scale-free structures, exhibited characteristics of self-organized critical (SOC) systems. This model, originally introduced to simulate real forest fires, has served as a paradigm for SOC systems and is referred to as the forest-fire (FF) model. In their investigations of the FF model, Grassberger and Kantz [2] found that unless the system was finely tuned, a characteristic time scale, inversely proportional to the tree growth probability $p$, emerged. So, in a strict sense, the FF model does not not display true SOC behavior which should, by definition, be free of any tuning. In an effort to overcome this failing, Drossel and Schwabl (DS) [3] introduced a lightning strike rule, which allowed the possibility of trees catching fire spontaneously with some (small) probability $f$. The ensuing result was that in the limit of low tree growth and random fire probabilities, this new (DSFF) model displayed SOC behavior. The downside of introducing such a modification are the conditions that both the tree growth $p$ and $f / p$ should tend to zero.

In this paper, we show that running a forest-fire model simulation on a lattice network, which includes some longrange interactions in addition to the local interactions of the lattice, results in scale-free structures. In addition to being fully deterministic and without recourse to random events as in the DSFF model, such a model has the added benefit of sitting on a network which may be used to simulate other phenomena; for example, the spread of disease in human populations.

\section{THE SMALL-WORLD FOREST FIRE (SWFF) MODEL}

Mimicking real world networks was the aim of Watts and Strogatz $[4,5]$ when they introduced the small-world (SW) network model. They developed real world network graphs which were neither constrained to have only local connections nor were just a random set of connections. Instead, it had long-range (randomly wired) connections between locally connected networks. This was, in effect, a network

*Email address: ig224@cam.ac.uk somewhere between a regular lattice with nearest neighbor connections and a random graph. The number of such connections in a network is characterized by the connection probability $q$.

There are different ways in which one can effect the longrange connections (shortcuts) between local networks in order to construct a small-world network. In their original paper, Watts and Strogatz [4] dealt with a one-dimensional (1D) ring lattice with nearest neighbor and next nearest neighbor connections. This network was then rewired by connecting randomly selected start and end points on the network (vertices of the graph) and simultaneously removing a connection from these vertices to their local neighbor. If one were to extend this approach to the FF model on a twodimensional square grid, the removal of the local connections would have a detrimental effect on the fire spreading potential which of course depends on these connections. It is therefore desirable to construct a different way of including the long-range connections or short cuts.

We considered two different approaches and for each, we calculated two measures which are used to characterize SW networks [4]. The path length $L$ is the shortest path connecting two vertices, averaged over all pairs. It is important to note that it is not a measure of the spatial distance between vertices (except in the special case of a regular graph) but is instead the smallest number of connections between the two vertices. $L$ is a global property of the graph since it measures some average communication between all pairs of vertices. It is therefore a function of the connection probability and, in particular, as $q$ is increased, the normalized path length $L(q) / L(0)$ would be expected to show a sudden decrease, reflecting the onset of SW behavior. The other measure is the clustering coefficient $C$, which is a local property of the graph and is concerned with the direct connections of the vertices.

Most SW networks are concerned with rewiring regular graphs or modifying random graphs in some way. The clustering coefficient is usually defined as the ratio of fully connected three node neighborhoods to the ratio of all three node neighborhoods. Since this quantity is trivial for our network (we almost never have connected triangles and always have connected squares), another measure of clustering, a local property of the graph, is required. We found it convenient to define the clustering coefficient as proportional to the inverse of the "lattice distance" (that is the number of steps to get from one point to another without using short- 
cuts). In this way, $C(q)$ measures a local property, i.e., how far away its neighbors are, and decreases as the number of shortcuts in the network increases.

The normalized coefficient $C(q) / C(0)$ is also expected to decrease once the small-world regime is entered. The smallworld regime is characterized by a small path length compared to that in the random graph and correspondingly high clustering of the lattice, i.e., a graph is SW when $L$ becomes significantly smaller than $C$. Thus, in the SW regime the difference measure, $D(q)=C(q)-L(q)$, and the ratio measure, $R(q)=C(q) / L(q)$, both become large.

The Newman-Watts algorithm. In the Newman and Watts procedure [6], a vertex chosen at random has a probability $q$ of being the starting point of a shortcut connection. The same is true for the ending point of a shortcut; thus each site has zero or one shortcut. Then, for each shortcut starting point, an end point is chosen at random and a connection is made. However, no vertices are deleted. The distribution of connections per site $P$ is $\delta$ distributed. So for $x$ connections,

$$
P(x)=(1-2 q) \delta_{4, x}+2 q \delta_{5, x} .
$$

To determine the range of $q$ values for which such graphs are SW in character, we calculated $L(q)$ and $C(q)$. The algorithm for obtaining $L(q)$ is as follows.

(i) Calculate the lattice distance or number of steps, $\mid x_{2}$ $-x_{1}|+| y_{2}-y_{1} \mid$, between the start $\left(x_{1}, y_{1}\right)$ and end $\left(x_{2}, y_{2}\right)$ points.

(ii) Search a $5 \times 5$ neighborhood of vertices around the start point and list all the shortcuts and find all the relevant connections.

(iii) Find the shortcut that brings the start and end points closer, taking into account the number of steps required to reach the shortcut from the start point.

(iv) If this route is closer than $\left(\left|x_{2}-x_{1}\right|+\left|y_{2}-y_{1}\right|-2\right)$ to the end point, move to the other end of the shortcut noting the number of steps taken.

(v) If there are no useful shortcuts, move closer to the end point by moving one step in the $x$ or $y$ direction.

(vi) Repeat until $\left(x_{1}, y_{1}\right)=\left(x_{2}, y_{2}\right)$.

(vii) Then, $L$ is simply the number of steps taken.

This procedure was repeated for all pairs of vertices satisfying the condition that $\left(\left|x_{2}-x_{1}\right|+\left|y_{2}-y_{1}\right| \geqslant 3\right)$ and then averaged. The minimum path length is then normalized with respect to the path length for the regular graph, $L(0)$. It may be noted that this algorithm does not necessarily find the absolute minimum path length each time, but the probability of finding a path length close to the absolute minimum is large.

The clustering coefficient $C(q)$ was obtained using an algorithm based on the one prescribed by Hayes [7]. The procedure is given as follows: (i) List the connections of a vertex (including the four nearest neighbors); (ii) sum the lattice distances $\left|x_{i}-x_{1}\right|+\left|y_{i}-y_{1}\right|$ of all the connections; (iii) divide the number of connections by the sum of the lattice distances; (iv) repeat over all vertices and take the average.

$L(q) / L(0)$ and $C(q) / C(0)$ were thus determined for a 2D square lattice of $128^{2}$ sites and the results for $q \leqslant 0.2$ are

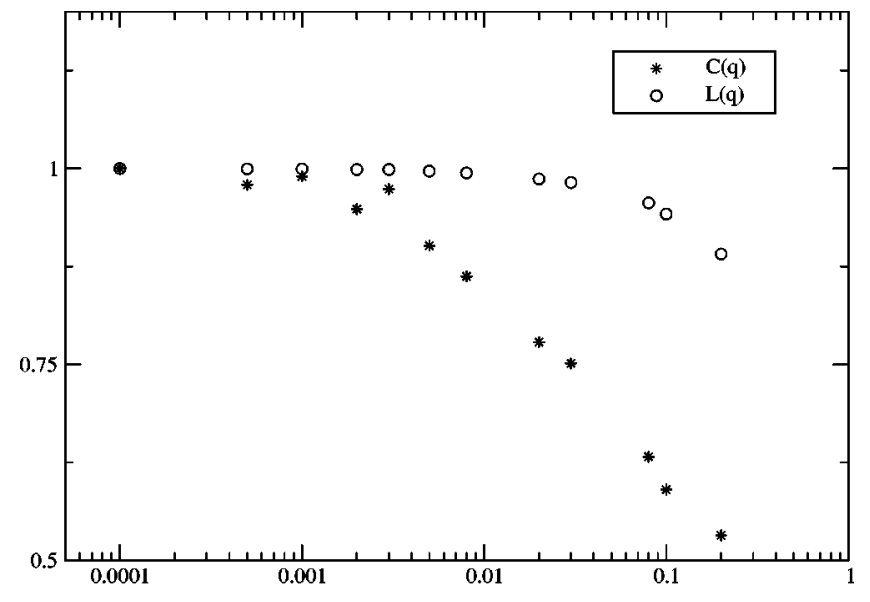

(a)

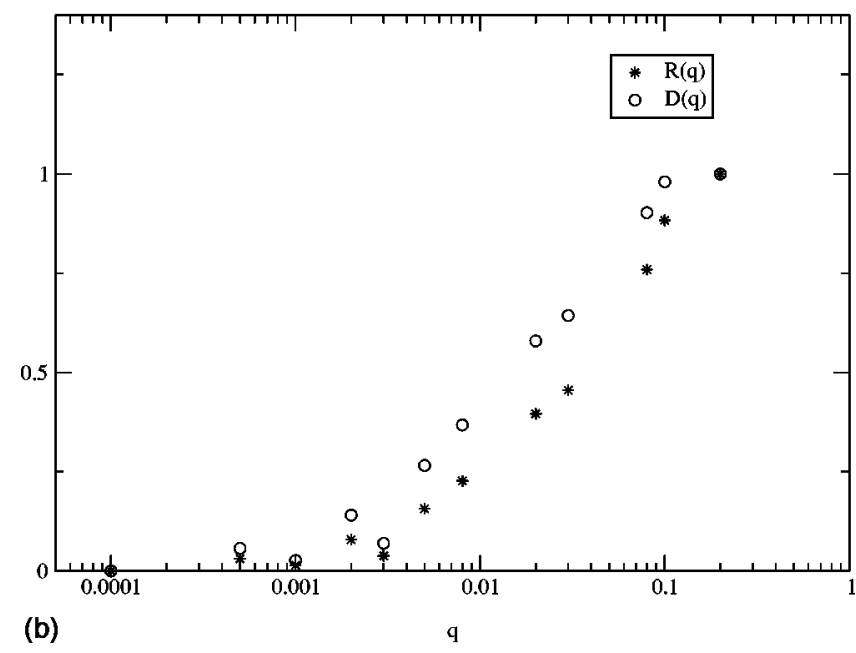

FIG. 1. (a) The normalized $L(q)$ and $C(q)$ and (b) the normalized $D(q)$ and $R(q)$ on a regular square graph using the NewmanWatts rewiring procedure.

shown in Fig. 1(a). The decrease in $L(q) / L(0)$, reflecting the onset of the SW regime is found to occur around $q$ $\sim 0.02$. This can be more clearly seen in the variation of the difference and ratio measures, $D(q) / D_{\max }$ and $R(q) / R_{\max }$, normalized to their maximum values [Fig. 1(b)]. Both of these quantities become large when $q \sim 0.005$. Although Watts and Strogatz found the onset of SW behavior for $q$ $\sim 0.002$, this is probably just a consequence of the increased connectivity and reduced dimensionality of their network.

The Kleinberg algorithm. In order to counter some of the difficulties encountered in carrying out the calculation of $L(q)$ in the Newman-Watts and the Watts-Strogatz procedure, Kleinberg [8] suggested a different method of reconnecting the network. In this approach, for each randomly chosen start point, the end points are randomly chosen from a power law probability distribution based on the lattice distance. By letting the probability of making a shortcut of distance $s$ decrease as a polynomial of order $r$ with increasing distance, this method of rewiring imprints a geographical structure onto the lattice thereby invoking the Milgram [9] navigational scheme. Then, for $r=2$, the minimum path length can be found using a decentralized algorithm in which 
only local information is available, given as follows.

(i) Choose start and end vertices of a path.

(ii) At each step, move from the present vertex (via a neighbor or a shortcut) to the vertex that is the smallest lattice distance away from the end vertex.

Because of the local nature of the search, it turns out that it is very much easier to compute the path length in this approach compared to that in the Newman-Watts method. However, with this scheme, the normalized path lengths and cluster sizes as a function of $q$ do not display SW characteristics until $q \sim 0.2$. As this is much too large a degree of rewiring and therefore unlikely to correspond to any realistic system, the Newman-Watts scheme was the one adopted in constructing our small-world network.

Our small-world forest-fire model was constructed by applying the DSFF model [3] to the small-world network rewired according to the Newman-Watts procedure. With this rewiring the DSFF model is modified to include the new rules.

(i) A shortcut array, in addition to the grid on which the forest-fire rules are run, is defined.

(ii) If a site is on fire, trees connected to it, either locally or through a shortcut connection, are set to catch fire at the next iteration.

(iii) The lightning strike probability $f$ is set to zero.

In order to determine whether the system has evolved to a SOC state, we determined the two measures (critical exponents) identified by Clar, Drossel, and Schwabl [10] as important in describing the structural features of the forest in its SOC state. The values of these measures may be used to define the universality class of the system. In their study, the exponent $\tau$ which governs the distribution $D(s)$ of tree clusters of size $s, D(s) \sim s^{-\tau}$, was found to vary from $\sim 2.1$ (for spatial dimension $d=2$ ) to $\sim 2.5$ (for $d \geqslant 6$ ). It may be noted that for $d \geqslant 6$, all the critical exponents in this model are independent of $d$. The second important critical exponent $\mu$ measures how the linear size of a cluster $R(s)$ depends on the cluster size through the scaling relation $R(s) \sim s^{1 / \mu}$. For $d=2$, it was found that $\mu=1.96$, indicating that though the cluster shape is statistically scale invariant, the clusters are fractal structures with capacity dimension equal to $\mu$. So any modification of the DSFF model must also result in power laws whose existence is required in order that it may be said to be a SOC system.

\section{MEAN FIELD THEORY FOR THE SWFF MODEL}

Following the approach of Christensen et al. [11] and Jensen [12], if we replace the individual site variables on the lattice network by the average densities $\rho_{e}(n), \rho_{f}(n)$, and $\rho_{t}(n)$ representing the empty, fire, and tree occupied states at iteration $n$, the evolution of these density distributions is described by the following mappings:

$$
\begin{gathered}
\rho_{e}(n+1)=\rho_{e}(n)-p \rho_{e}(n)+\rho_{f}(n), \\
\rho_{t}(n+1)=\rho_{t}(n)+p \rho_{e}(n)-q \rho_{f}(n) \rho_{t}(n)-m \rho_{f}(n) \rho_{t}(n),
\end{gathered}
$$

$$
\rho_{f}(n+1)=q \rho_{f}(n) \rho_{t}(n)+m \rho_{f}(n) \rho_{t}(n) .
$$

Here, $p$ represents the tree growth probability, $q$ the connection probability, and $m$ is the coordination number of the lattice (i.e., the number of nearest neighbors for $q=0$ ) which for a square lattice is 4 . Because each fire site becomes an empty site at the next iteration whereas an empty site has a probability $p$ of becoming a tree site, these contribute to the change in the empty site density in Eq. (1). The latter contribution also results in a corresponding increase in the tree density. The decrease in $\rho_{t}$ comes about as trees catch fire if they are connected to a fire site, either through a short range (nearest neighbor) connection or through a shortcut at the preceding iteration. Since these contributions require a tree site to be connected to the appropriate fire site, they include the product term $\rho_{f}(n) \rho_{t}(n)$. Finally, the fire site density is obtained from the constraint that all the changes must add to zero. In addition to these equations we have the further constraint that all sites must be empty, occupied by a tree or by a fire site. This leads to the sum rule

$$
\rho_{e}(n)+\rho_{t}(n)+\rho_{f}(n)=1 \text {. }
$$

In order to solve these equations, we follow the approach of Christensen et al. [11] and write the difference equations (1)-(3) above as differential equations

$$
\begin{gathered}
\dot{\rho}_{e}=-p \rho_{e}+\rho_{f}, \\
\dot{\rho}_{t}=p \rho_{e}-q \rho_{f} \rho_{t}-m \rho_{f} \rho_{t}, \\
\dot{\rho}_{f}=-\rho_{f}+q \rho_{f} \rho_{t}+m \rho_{f} \rho_{t} .
\end{gathered}
$$

Then, substituting for $\rho_{e}$ from Eq. (4) in Eqs. (6) and (7), we get

$$
\begin{gathered}
\dot{\rho}_{t}=p\left(1-\rho_{f}-\rho_{t}\right)-(q+m) \rho_{f} \rho_{t}=F\left(\rho_{f}, \rho_{t}\right), \\
\dot{\rho}_{f}=-\rho_{f}+(q+m) \rho_{f} \rho_{t}=G\left(\rho_{f}, \rho_{t}\right) .
\end{gathered}
$$

To find the stationary solutions to these coupled equations, we put $F=G=0$. This gives the fixed points of the SWFF model in the phase space of the fire and tree densities, viz.,

$$
\begin{gathered}
\rho_{t}^{*}=\frac{1}{m+q}, \\
\rho_{f}^{*}=p \frac{\left(1-\frac{1}{m+q}\right)}{1+p}=p\left(\frac{1-\rho_{t}^{*}}{1+p}\right) .
\end{gathered}
$$

The behavior of the system in the vicinity of the fixed points is determined by the eigenvalues $\lambda$ of the determinantal equation 


$$
\left|\begin{array}{cc}
\frac{\partial F}{\partial \rho_{t}}-\lambda & \frac{\partial F}{\partial \rho_{f}} \\
\frac{\partial G}{\partial \rho_{t}} & \frac{\partial G}{\partial \rho_{f}}-\lambda
\end{array}\right|=0 .
$$

The eigenvalues are simply the roots of the equation $\lambda^{2}$ $+B \lambda+C=0$, where

$$
B=p\left(1+\frac{m+q}{1+p}\right)
$$

and

$$
C=p\left\{1+(m+q)\left(\rho_{f}-\rho_{t}\right)\right\}+(m+q) \rho_{f} .
$$

Since we are mainly interested in the behavior around the fixed points, we substitute the general fire and tree densities for their values at the fixed points. Then, in the limit of slow driving, $p \rightarrow 0$,

$$
B=p\left(\frac{m+q}{1+p}\right)
$$

and

$$
C=p(m+q-1)
$$

Thus both coefficients tend to zero as $p \rightarrow 0$. However, both solutions are complex since $B^{2}$ goes to zero as $p^{2}$ while $C$ goes to zero as $p$. Also, since $B$ is always positive, the real parts of the solution are negative. Thus the trajectory in phase space around the critical point is an attractive spiral. Note that this is so for $p \rightarrow 0$ irrespective of the reconnection probability $q$. In the DSFF model, the fixed point is an attractive spiral for $f / p \rightarrow 0$ and $p \rightarrow 0$. So the SWFF model, in the mean field approximation, possesses an attractive critical point similar to that found in the DSFF model, but with only one requirement that $p \rightarrow 0$. This is akin to requiring only a slow driving of the system.

In fact it can easily be shown that the system always possesses a nontrivial stable spiral at $\left(\rho_{f}^{*}, \rho_{t}^{*}\right)$. Slow driving is not required to show SOC in the mean field interpretation. In this sense the mean field theory (MFT) does not make any predictions about which parameter regime we can expect to observe SOC in. We will find a steady state at nonzero $\left(\rho_{f}^{*}, \rho_{t}^{*}\right)$, but we need to resort to simulation to say anything about the SOC (or otherwise) properties of this steady state.

\section{RESULTS OF SIMULATIONS}

Simulations of the SWFF model on a $128 \times 128$ square lattice were carried out for the rewire parameter $q$ varying between 0 and 0.3. $q$ was restricted to be less than 0.3 because too high a reconnection probability would result in far too many long-range interactions to be interesting. It was however necessary to study $q$ at around 0.2 as it is for 0.02 $<q<0.2$ that the graph is well within the small-world regime.

We explored the whole of $(p, q)$ space and looked for
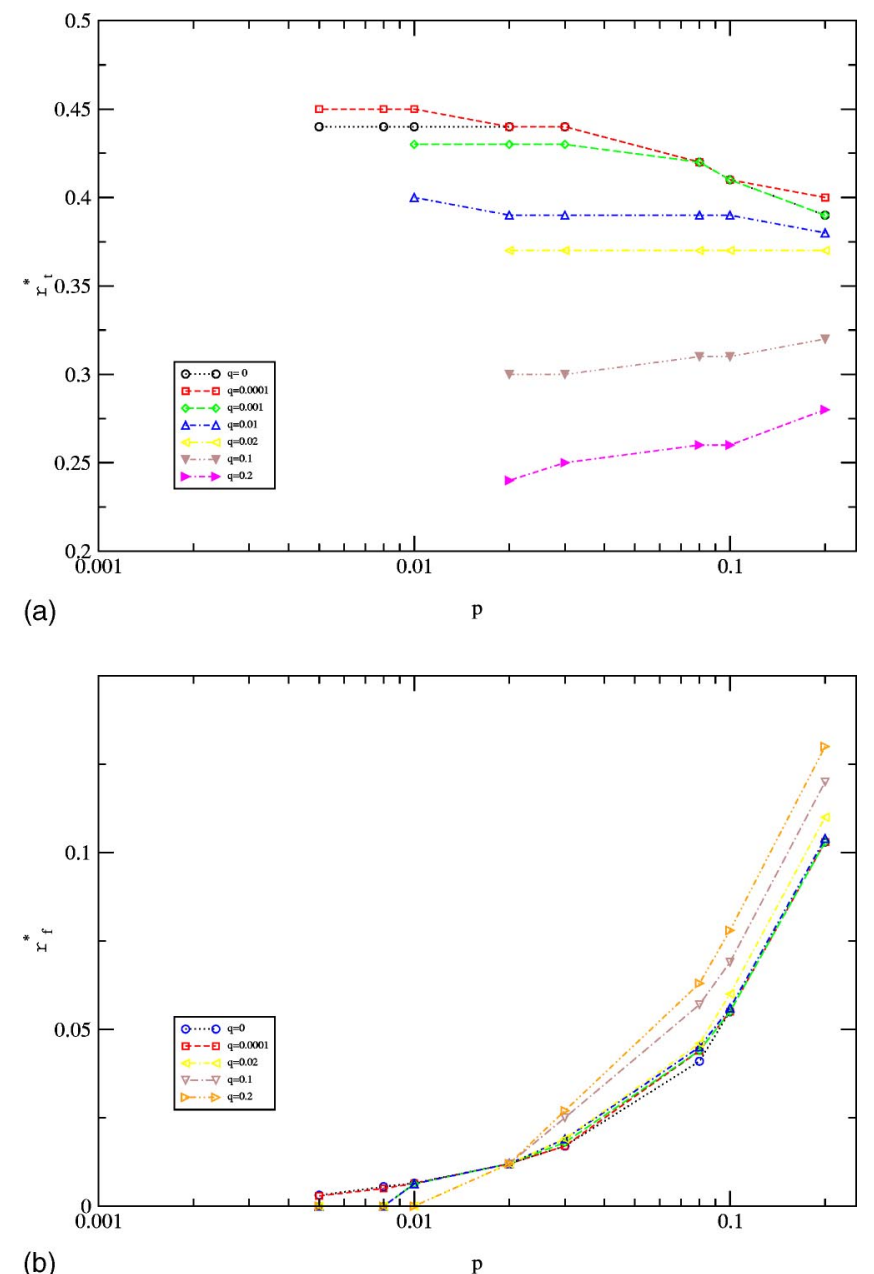

(b)

$\mathrm{p}$

FIG. 2. The variation of the steady state (a) tree and (b) fire densities with the driving parameters $p$ for different values of the rewire parameter $q$.

SOC (or other) behavior at various points on the phase plane. Simulations were carried out on a $256^{2}$ lattice, and the steady state fire and tree densities were recorded. For large $q$ and small $p$ the system still evolves to a nonzero steady state, but the attracting spiral becomes very weak. Since the system is finite, the amplitude of the spiral from an arbitrary initial condition is large and the system tends to become trapped in a state in which there are no fires.

Interesting behavior occurs above $q=0.02$. We look for a change in the "order parameter" ( $\rho_{f}^{*}$ or $\rho_{t}^{*}$ ) of the system as $q$ increases [14]. There is no abrupt change in order parameter, but the relation between $\rho_{f}^{*}, \rho_{t}^{*}$, and $p$ changes at $q$ $\sim 0.02$. Figure 2(a) shows this effect: Below $q=0.02$ tree density increases with $p$, while above $q=0.02$ it decreases. This is due to a jump in fire density in this region [Fig. 2(b)], but only for $p>0.02$.

The system therefore changes qualitatively in the phase space region $q \geqslant 0.02, p \geqslant 0.02$. We can investigate this regime by finding the relevant power law exponents. Note that MFT predicts no change in the tree density for a given $q$, and that the fire density increases monotonically with both $p$ and $q$. 


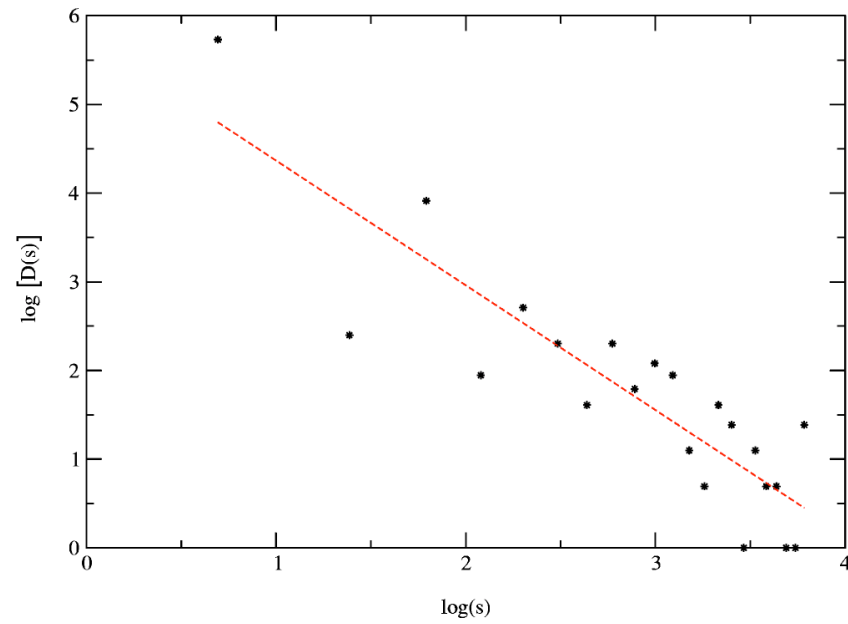

FIG. 3. Size distribution $D(s)$ of cluster sizes $s$ for the SW model for $q=0.3$ and $p=1$.

Although the mean field theory analysis was carried out in the small $p$ limit, there is no need to impose this constraint in simulations. Since the tree growth probability $p$ is associated with the strength of the driving of the system, a high value of $p$ corresponds to a strongly (over) driven system. Such a system can be viewed as one in which the probability of trees growing on empty sites is very high. Alternatively, high $p$ corresponds to a model of a very dense system.

Taking these factors into account, two sets of simulations were carried out. In the first, $p$ was kept fixed at a relatively small value and $q$ allowed to vary between 0 and 0.3 . In the other set of simulations, $q / p$ was set to a constant low value and $q$ allowed to vary in the range. As $p$ is always less than 1 , the simulations were carried out for $q / p=0.02$, with $q$ $\leqslant 0.2$ and for $q / p=0.1$, with $q \leqslant 0.1$. In all of the simulations both the cluster size and the linear size distributions displayed very good scaling behavior.

The scatter is a consequence of the onset of spiral shaped structures, which are reminiscent of the "self-organized genetic drift" (see, for example, Schroeder [13]) when the interaction between neighboring sites results in pattern formation. The presence of patterns are, of course, indicative of some type of periodic or quasiperiodic behavior. These results may be explained by noting that with this fast driving, the evolution of the system follows a deterministic, periodic pattern with trees growing on empty sites, then catching fire and leaving empty sites only to start again. This is manifest in the spatial distribution of sites, which although displaying a range of cluster sizes, appear spiral shaped and are dominated by fire resulting from a three-step cycle. The spiral shapes ensuing from this fast driven system are quite complex as they tend to start at randomly distributed shortcut points.

Even in the limit of high driving, $p=1$ and high reconnection probability, $q=0.3$, the results scaled well enough to make it possible to extract values of the critical exponents, $\tau$ and $\mu$ (Figs. 3 and 4). However, it is unclear as to why the spiral structures should display scaling since they are not fractal.

The results for smaller $p$ values show much less scatter.

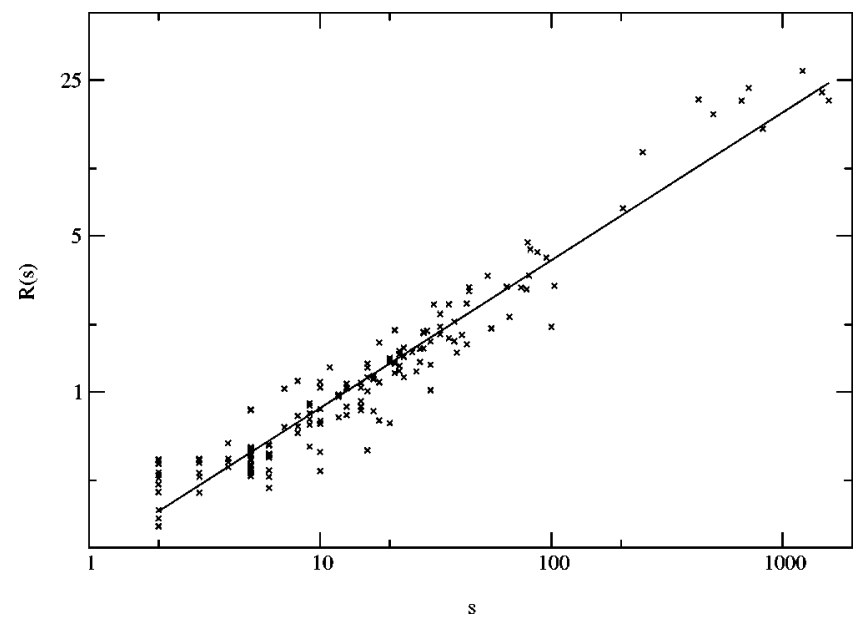

FIG. 4. The variation of the linear size $R(s)$ with clusters of size $s$ for $q=0.3$ and $p=1$.

As expected, in the limit $p, q \rightarrow 0, \tau$ was found to be $\sim 2.1$, and $\mu \sim 1.8$ in good agreement with the results obtained for the DSFF model.

$p=0.03$ simulations. The averaged cluster distribution exponent for $0.002 \leqslant q \leqslant 0.2$ and $p=0.03$, is shown in Fig. 5 . There is no discernable variation of $\tau$ with $q$ and it remains relatively constant around $\sim 2.1$. This is in keeping with mean field theory analysis which indicates that the SWFF model should achieve criticality in the limit $p \rightarrow 0$ independent of the reconnection probability. A plausible explanation for this is that, in the limit of slow driving, there is a buildup of correlations between shortcuts as well as on the regular grid network. A shortcut whose vertices are both in the center of clusters will not cause a fire to start. Thus, in a qualitative sense, increasing the shortcut density does not affect the growth of the few large scale structures that are required in order for the distribution to follow a power law (as long as the "random" regime is not entered) and $\tau$ is not greatly different from the DSFF value.

The critical exponent $\mu$ also shows no general trend with increasing $q$ but there is a little more scatter and $\mu$ is in the

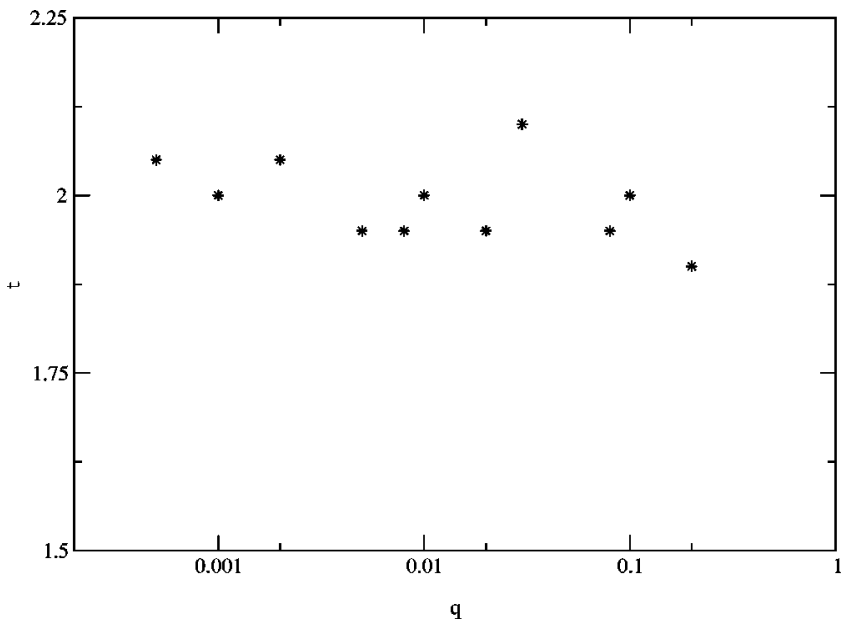

FIG. 5. The cluster size distribution exponent $\tau$ vs the reconnection probability $q$ for $p=0.03$. 


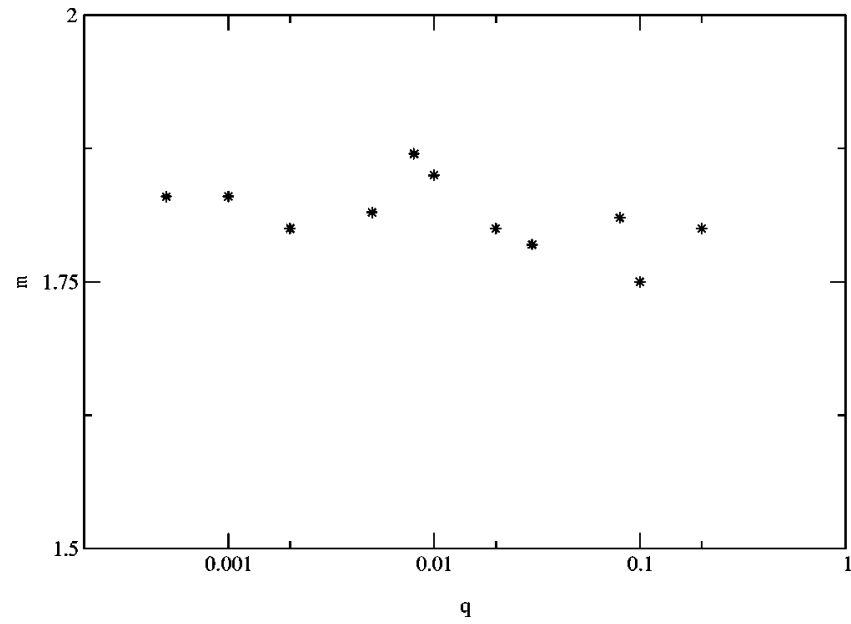

FIG. 6. The linear size distribution exponent $\mu$ vs the reconnection probability $q$ for $p=0.03$.

range $1.77-1.87$ (Fig. 6). Thus, the $p=0.03$ simulations seem to be SOC, independently of $q$. To investigate the phase space where new behavior might take place, a different set of simulations was carried out.

Constant $q / p$ simulations. With the algorithm described above, a qualitatively different regime is entered as $q$ approaches 0.02 . Since the speed of driving (the rate of energy input or in this case tree growth probability) is an important factor in characterizing the system, it is pertinent to consider separately the evolution of the system in the case of fast and moderate driving of the system.

Thus, setting $q / p=1 / 50$, the $\mathrm{SW}$ regime is encountered only for $p$ becoming quite large $(\sim 1)$, corresponding to fast driving. In these simulations, the cluster size exponent $\tau$ was found to change appreciably with the reconnection probability (Fig. 7). Specifically, as $q$ approaches the SW regime, $\tau$ decreases quite sharply, falling from its initial value of around $2.1(0.01>q>0.0005$ and $0.2>p>0.025)$ down to about 1.3. This is exactly the region in which there is a change in the behavior of the order parameters.

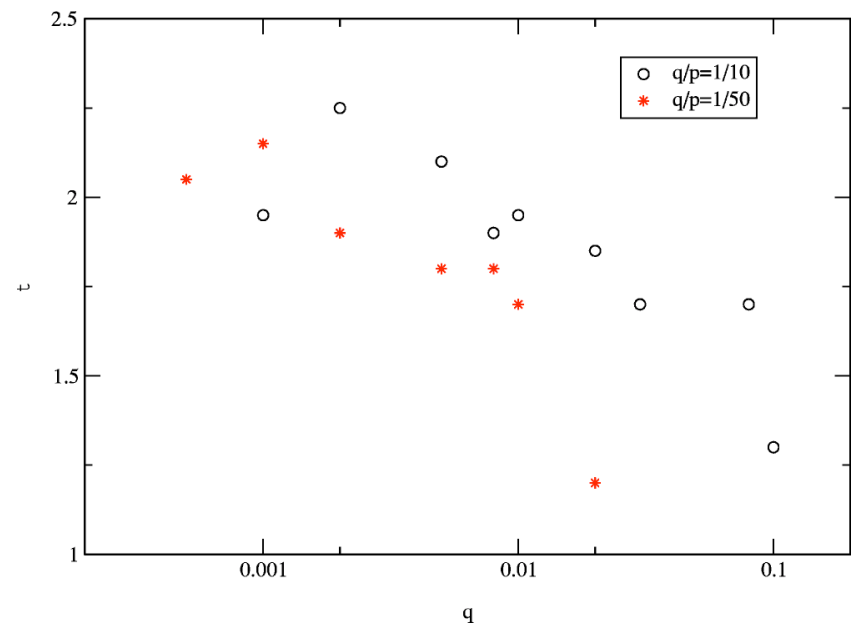

FIG. 7. The cluster size distribution exponent $\tau$ vs the reconnection probability $q$ for $q / p=1 / 50$ and $q / p=1 / 10$.

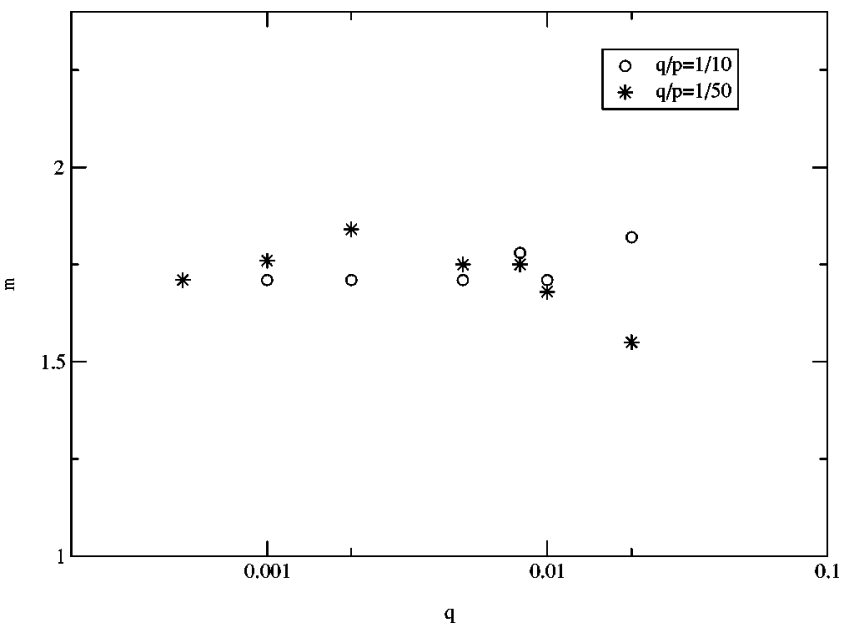

FIG. 8. The linear size distribution exponent $\mu$ vs the reconnection probability $q$ for $q / p=1 / 50$ and $q / p=1 / 10$.

The linear size exponent $\mu$ also experiences a sharp fall as $q \rightarrow 0.02$ (Fig. 8). For values of $q$ greater than 0.02 , there is considerable fluctuation in $\mu$ values which is just a reflection of the high degree of scatter in the $R(s)$ distributions. This is in keeping with the presence of the aforementioned spiral shapes.

For $q / p=1 / 10$ the SW regime is entered into when $p$ is still relatively small, $p \sim 0.2$, and is therefore not overdriven. However, even in this instance, the distribution measure, $\tau$ decreases from its original value of 2.1 to about 1.7 (Fig. 7). Thus the decrease in $\tau$ as the model becomes SW persists even when $p$ is quite small. By contrast, the size measure $\mu$ displays no clear relationship with the reconnection probability and remains relatively constant (Fig. 8).

\section{DISCUSSION}

We have demonstrated that running the FF model on a network which has in-built long-range connections in addition to the normal nearest neighbor interactions results in a SOC system for slow driving and a "scale-free" system that has complex structures but cannot be considered SOC for strong driving.

We looked for a qualitative change in the behavior of the system in $(p, q)$ space. The tree density was found to decrease with increasing driving above $q=0.02$. There was a jump in fire density for $q>=0.02, p>=0.02$. This regime corresponds to larger driving-fires spread easily through new tree growth and an appreciable number of shortcuts. MFT does not predict these qualitative changes.

In the region where qualitatively different behavior takes place, different power law exponents for cluster size distribution and fractal dimension were found. This corresponds to a "phase transition" from the SOC state to a different self-organized state. This can be seen from the transition of cluster shape from fractals to spirals.

The strongly driven regime cannot be considered as SOC, rather it is a different type of self-organization caused by strong driving and a large number of shortcuts. 
Unlike the Drossel-Schwabl model this modified model is deterministic and shows complex behavior without any fine tuning. Both, the DSFF and SWFF models have different ranges of applications. Thus while the SWFF model would not be appropriate in modeling the evolution of real forest fires or of phenomena where there are random occurrences, the long-range connections makes it suitable for modeling the interaction of human populations, where the driving of the system is not necessarily slow.

In particular, it may be adapted to study the spread of disease in immunized communities. In studying such systems, it is the change in the universality class or the breakdown of SOC which are of importance as they would suggest a possible breakdown of the dynamic equilibrium of the system.
[1] P. Bak, K. Chen, and C. Tang, Phys. Lett. A 147, 297 (1990).

[2] P. Grassberger and H. Kantz, J. Stat. Phys. 63, 685 (1991).

[3] B. Drossel and F. Schwabl, Phys. Rev. Lett. 69, 1629 (1992).

[4] D.J. Watts and S.H. Strogatz, Nature (London) 392, 440 (1998).

[5] D. J. Watts, Small Worlds (Princeton University Press, Princeton, 1999).

[6] M.E.J. Newman and D.J. Watts, Phys. Rev. E 60, 7332 (1999).

[7] B. Hayes, Am. Sci. 88, 104 (2000).

[8] J. Kleinberg, Cornell Computer Science Technical Report No. 99, 1999 (unpublished).
[9] S. Milgram, Psychology Today 1, 61 (1967).

[10] S. Clar, B. Drossel, and F. Schwabl, Phys. Rev. E 50, 1009 (1994).

[11] K. Christensen, H. Flyvbjerg, and Z. Olami, Phys. Rev. Lett. 71, 2737 (1993).

[12] H. J. Jensen, Self-Organized Criticality (CUP, Cambridge, 1998).

[13] M. Schroeder, Fractals, Chaos and Power Laws (Freeman, 1990).

[14] S.Y. Huang, X.W. Zou, Z.J. Tan, and Z.Z. Jin, Phys. Rev. E 67, 026107 (2003). 